\title{
On Dynamic Consensus Processes in Group Decision Making Problems
}

\author{
I.J. Pérez ${ }^{\mathrm{a}}$, F.J. Cabrerizo ${ }^{\mathrm{b}}$, S. Alonso ${ }^{\mathrm{c}}$, Y.C. Dong ${ }^{\mathrm{d}}$, F. Chiclana ${ }^{\mathrm{e}}$, E. Herrera-Viedma ${ }^{\mathrm{b}}$ \\ ${ }^{a}$ Dept. of Computer Sciences and Engineering, University of Cadiz, ignaciojavier.perez@uca.es, Cadiz, \\ Spain \\ ${ }^{b}$ Dept. of Computer Science and Artificial Intelligence, University of Granada, cabrerizo@decsai.ugr.es, \\ viedma@decsai.ugr.es, Granada, Spain \\ ${ }^{c}$ Dept. of Software Engineering, University of Granada, zerjioi@ugr.es, Granada, Spain \\ ${ }^{d}$ Dept. of Business School, Sichuan University, ycdong@scu.edu.cn, Chengdu, China \\ ${ }^{e}$ Centre for Computational Intelligence, Faculty of Technology, De Montfort University, \\ chiclana@dmu.ac.uk, Leicester, UK
}

\begin{abstract}
Consensus in group decision making requires discussion and deliberation between the group members with the aim to reach a decision that reflects the opinions of every group member in order for it to be acceptable by everyone. Traditionally, the consensus reaching problem is theoretically modelled as a multi stage negotiation process, i.e. an iterative process with a number of negotiation rounds, which ends when the consensus level achieved reaches a minimum required threshold value. In real world decision situations, both the consensus process environment and specific parameters of the theoretical model can change during the negotiation period. Consequently, there is a need for developing dynamic consensus process models to represent effectively and realistically the dynamic nature of the group decision making problem. Indeed, over the past few years, static consensus models have given way to new dynamic approaches in order to manage parameter variability or to adapt to environment changes. This paper presents a systematic literature review on the recent evolution of consensus reaching models under dynamic environments and critically analyse their advantages and limitations.
\end{abstract}

Keywords: Group decision making; dynamic decision support systems; consensus process; multi period decision making; adaptive consensus models.

\section{Introduction}

Group Decision Making (GDM) is usually described as a best alternative(s) selection process of selection from a given set of feasible options based on the opinions of a group of people, frequently referred to as experts. This process is of importance not only when the decision consequences affect a group of people, but also when the decision itself can be improved, that is to make better decisions, by involving more people in the decision process $[5,9,30]$.

Ideally, unanimous decisions, i.e. total agreement on the decision by all experts, are aimed at, although it is not really necessary. Indeed, the majority rule is frequently presented as a cornerstone of any democratic decision $[6,12]$. Nevertheless, different situations may require 
different rules. For example, the minority rule or the unique person rule could be more operative in order to save time and reduce cost in companies. Nowadays, though, it is more and more important to make decisions under the consensus rule, i.e. to make consensual decisions by reaching a general or widespread agreement among the involved people. As such, consensus reaching process has become an important research area in GDM problems $[6,17,23]$.

As mentioned above, full consensus is not necessary and even untenable in many real world situations. This could be a result of important differences between experts, which are inherent to their own knowledge and personal interests. Thus, consensus within GDM has been alternatively modelled following a 'softer' methodology for its measurement that allows a range possibilities from absence to total agreement. This soft consensus measures, which aims to be realistic and flexible, is based on the implementation of fuzzy set and logic concepts and tools $[4,6,12,24]$.

In practice, consensus reaching processes proceed in a convergent multistage way, where experts present their individual preferences at the beginning of the process and, while consensus level is not considered enough, they discuss, negotiate and bring positions closer by modifying their initial opinions [22]. It is, therefore, assumed that the individuals are prepared and willing to commit to those opinion changes. Sometimes there exists a particular person (or automated system) who acts like a moderator, being responsible of the process management until the experts reach agreement [1].

Decision problems require a careful analysis of the current environment conditions and characteristics of the problem to be solved. All decision problems do not take place in a static environment. In fact, when the current problem conditions or parameters vary during the discussion phase, the problem final solution could vary as well. Indeed, it is clear that many external and subjective factors could affect decision processes. As a consequence, the consensus process has been recently studied as a dynamic process and some researchers have focused on the incorporation of diverse dynamic parameters or variables to static consensus models. The purpose of this paper is to provide a comprehensive description of the current state of the art of dynamic consensus approaches and to analyse the following different new trends and challenges in this field:

- Classical Dynamic Consensus Approaches in which the consensus process, by definition, is an iterative and dynamic process and the experts' preferences can be modified until an agreement is reached by increasing the consensus level.

- Time Modelling Consensus Approaches present a new variable to model time $(t)$. Time modelling approach aims at predicting opinion dynamics in time (from $t$ to $t+1$ ) by using time series. Additionally, in multi-period decision making approaches, an expert's different preferences at different time periods are aggregated using new operators, such as the Dynamic Weighted Averaging (DWA) operator and its extensions.

- Dynamic Environment in Consensus Approaches. The consensus process environment can also change over time. Consequently, in order to reflect these changes in the consensus model model itself, the following diverse dynamic variables have been considered/analysed in the literature: i) dynamic alternatives, ii) dynamic experts, iii) dynamic expert's importance and iv) dynamic criteria. 
- Adaptive Consensus Approaches. The consensus model behaviour can also be dynamic. Feedback mechanism to generate recommendations, when present can also adapt dynamically with respect to: i) the collective consensus level (CL), ii) the individual consensus indexes (ICI) and iii) the expert's importance.

The rest of the paper is set out as follows. Section 2 presents preliminary concepts on consensus reaching processes and the research methodology used to define the systematic literature review. In Section 3, the different dynamic consensus reaching models, considered as main contributions in the literature review, are described. In Section 4 a discussion among the reviewed approaches is presented, and potential future research avenues proposed to improve the current methods. Finally, in Section 5 conclusions are drawn.

\section{Preliminaries}

The resolution of a GDM problem involves two main processes [22, 39]:

1. The consensus process that aims to support experts until a satisfactory level of agreement among them is reached.

2. The selection process based on the individual experts' opinions, which are usually fused into a collective opinion, from which a group decision is derived, mainly via the ranking of the different alternative.

This section presents preliminary information on consensus reaching processes in GDM problems. Then, the systematic literature review process carried out to complete the research described.

\subsection{Consensus reaching processes in GDM problems}

A consensus reaching process model can be described as a multi stage process (dynamic and iterative) that simulates real face to face discussion or negotiation processes between a group of experts. It relies on the assumption of experts' willingness to change their opinions or preferences, in several consensus rounds, in order to reach a collective agreement on a decision. It is not unusual for a moderator or facilitator person to support experts in reaching such agreement, which can be automated within an appropriate consensus support system.

To date, multiple consensus reaching models have been published, with quite a large number of them implementing soft consensus measures and fuzzy logic based rules to guide and control the process $[1,4,6,22,33]$. An analysis of these models reveals a working operation pattern or framework, which is graphically depicted in Figure 1 and described below.

(1) Before the start of the discussion process, experts are informed about the particular parameters of the problem and the different alternatives to choose the best ones from.

(2) Experts express their individual opinions or preferences on the alternatives by means of a preference representation format (ranking of alternatives, evaluations or pairwise comparison matrix). 


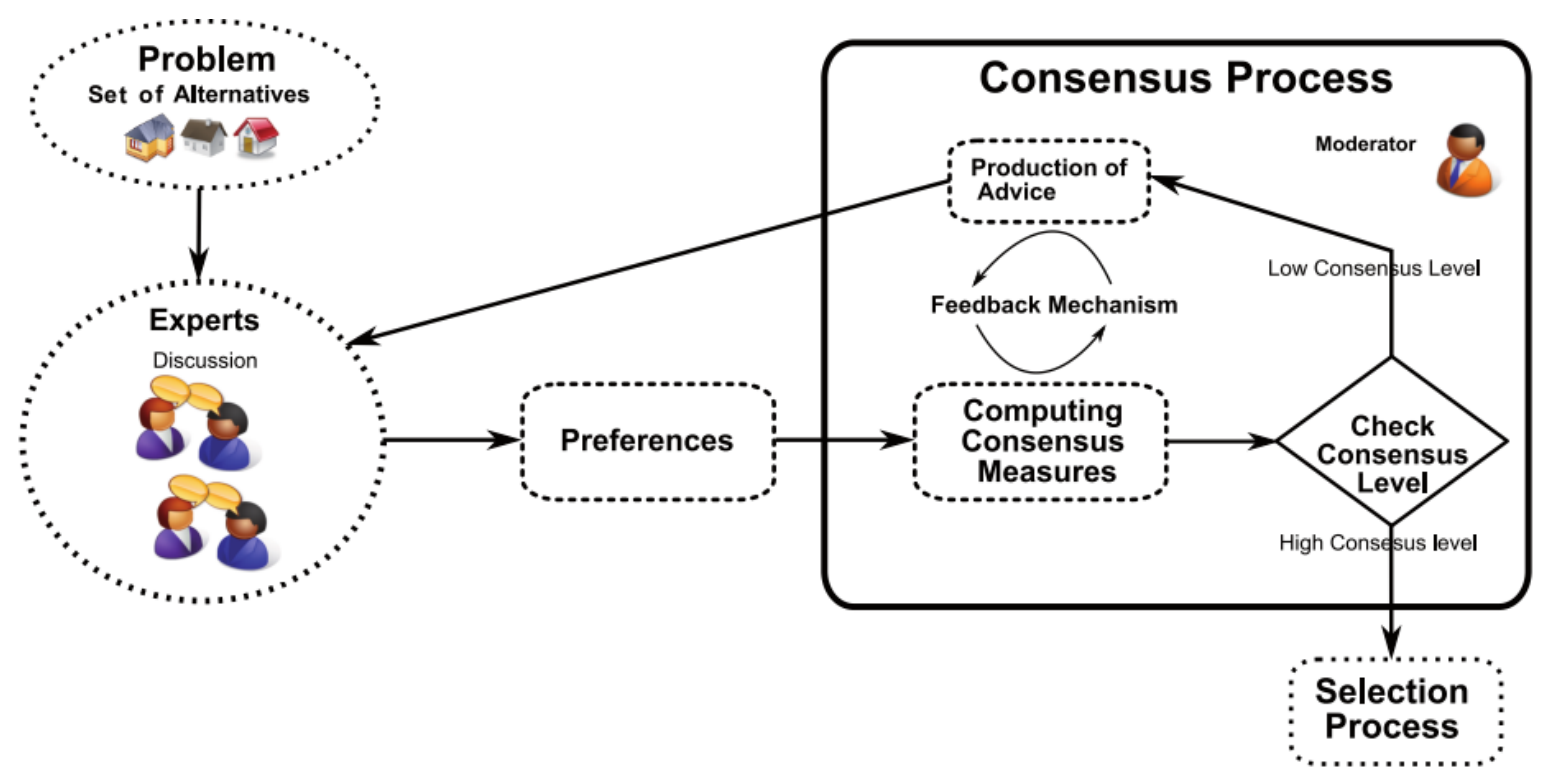

Figure 1: General Consensus Framework

(3) The moderator/system computes consensus measures based on how distant the individual preferences are. These measures are used to decide when the group consensus level reach is sufficient.

(4) If the group consensus level is satisfactory, the selection process is activated. Otherwise, a new discussion round is carried out. Most consensus models implement what is known as a feedback mechanism, which is used to feedback experts on how to improve the group consensus level.

(5) Some or all experts might change their opinions after the feedback information received, which will subsequently modify the group consensus. At this point, step (3) is activated again until the minimum group consensus threshold values is achieved or a maximum number of consensus rounds is reached.

It is worth noticing that in the above consensus framework (classical consensus approaches), decision making problem parameters (experts set, alternatives set, experts' importances, set of criteria etc.) remain fixed through the discussion rounds. In other words, the model configuration is set prior the first stage of the consensus process and it is not modified during the whole process. This static framework of consensus is too rigid in practice as there could be external factor, such as weather conditions, price fluctuations, alternatives and/or experts availability, that could happen and that would make the above framework impractical. To address this issue, recent approaches have been proposed that allow the variation of certain parameters and consequently the implementation of a dynamic structure in the above consensus framework. Consequently, a new generation of dynamic and adaptive consensus reaching models have been developed over the past few years. This paper present a review, analysis and classification of these new kind of dynamic consensus reaching models. 


\subsection{Consensus measures in GDM problems}

As per Laughlin [26], social decision scheme and social choice theory address the same fundamental problem, which it is the way in which a group of people combine or aggregate a distribution of member preferences in a collective decision subject to previously established parliamentary procedures and voting rules. Different rule procedures imply different group decision making methods, which range from directive to participatory methods. Methods are closer to the directive one when the decision is made by a limited number of decision makers in the group. For example, individual dominance method where a single person has the authority to make the final decision, or minority influence method that usually takes the form of decisions delegated from larger groups and made by sub committees [2]. On the other hand, methods are closer to the participatory one when every member of the group is involved in making the final decision. Examples of participatory methods are majority rule methods include a range of voting systems where the number of votes received by each alternative establish the final ranking, while consensus methods where a consensual agreement has to be reached by all group members. This paper focuses on this last group of participatory procedures, i.e. on procedures to reach consensus among a group of experts, and as such will present an analysis of different consensus measures of the current literature.

As mentioned before, consensus is usually defined as the total and unanimous agreement of all the experts in relation to the feasible alternatives [12]. This definition implies the existence of only two states of consensus (absence and total agreement), which translate into a hard consensus measurement $(\{0,1\})$ in accordance to classical logic. In any case, full and unanimous agreement might be non-realistic and unnecessary in practice. An alternative realistic approach to consensus measurement that extends the above hard/crisp measurement is preferred. Kacprzyc et. al. in [23] proposed the use of soft consensus measures based on fuzzy logic to quantify the level of consensus level to reflect the range of partial agreement states located between the mentioned absence and total agreement states. Soft consensus measures are based on the concept of coincidence among experts' preferences, and a study by Cabrerizo et al. [6] identified three different coincidence criteria:

1. Strict coincidence among preferences. This consensus approach is based on the concept of equality, and it assumes only two possible coincidence values $\{1,0\}$ for when preferences are equal or not, respectively. The advantage of this approach is that the computation of the consensus degrees is simple and easy. However, the drawback is that the consensus degrees obtained do not reflect the real consensus situation, with similar preference values but different are treated equally to very dissimilar preference values.

2. Soft coincidence among preferences. This approach is based on the concept of similarity rather than equality. Thus, a similarity function is define, based on a distance function between preference values, which allows for a gradual computation of the coincidence concept in $[0,1]$, which resembles better the real consensus situation. This criteria is computationally is more expensive than the strict coincidence criterion.

3. Coincidence among solutions. The advantage of this approach is that the consensus state is evaluated by comparing the individual solutions each expert would obtain without considering other experts' preferences. This approach is even more expensive computationally than the soft coincidence criterion. 
From the above, it is clear that consensus measures are based on the implementation of a similarity (distance) function. As such, it s worth mentioning the study by Del Moral et al. [12] on five of the most commonly used distance functions in modelling soft consensus measures: Manhattan, Euclidean, Cosine, Dice and Jaccard.

\subsection{Systematic literature review process}

In order to carry out a systematic review of publications on dynamic consensus reaching approaches currently available in public research repositories, the guidelines proposed by Kitchenham et al. in [25] have been followed. The two main databases of bibliographical references in the world have been used: Web of Sciences (Clarivate Analytics) and Scopus (Elsevier).

With the purpose of finding the relevant papers and excluding irrelevant ones on topics such as voting systems or approaches that do not use the concept of consensus to guide the decision making process, the terms "dynamic", "changeable", "multi period" and "adaptive " were entered simultaneously with "group decision making" or "GDM" and "consensus". In each case, papers not related to dynamic consensus models were excluded. The references section of a considered relevant paper was also inspected to include some of the references when appropriate.

\section{Analysis and classification of current dynamic consensus reaching approaches}

Dynamic consensus approaches can be classified in one of the following four main categories or methodologies: Classical Dynamic Consensus Approaches; Time Modelling Consensus Approaches; Dynamic Environment in Consensus Approaches; and Adaptive Consensus Approaches.

\subsection{Classical Dynamic Consensus Approaches}

Traditionally, the consensus reaching problem is theoretically modelled as a multi stage negotiation process, i.e. an iterative process with a number of negotiation rounds, which ends when the consensus level achieved reaches a minimum required threshold value. Classical approaches focused their attentions on the iterative feature of the consensus process, and in particular on the modification of the experts' preferences to increase their level of consensus. This is mainly achieved by the design and building of appropriate feedback mechanisms to provide recommendations to experts based on their current state within the consensus process, which in practice means replacing the physical moderator with a virtual moderator. Other contributions on this category aimed at speeding the process by proposing the use of new technologies such as web sites or smartphones applications to support the process. In this way, the experts can resume the process and change their preferences at anytime and anywhere.

\subsubsection{Change of preferences}

Since the late 70s, several approaches on how groups reach agreement via the changing of experts's preferences when the current level of consensus is not sufficient have been carried out $[4,6,12,38,45,50]$. 
The first authors who appeared to use the term "dynamic" to refer to the changing of preferences at each consensus process iteration were Fedrizzi et al. in 1999 [17]. Indeed, in this work the authors modelled the consensus process as a dynamical network that combines the minimization of a soft measure of collective dissensus, $V$, and an individual inertial mechanism which tries to model opinion changing aversion, $U$. The full cost function, $W$, was then computed as function of $V$ and $U$. Finally, the current iterative dynamism of the consensual network corresponds to a parallel gradient descent scheme for the full cost function $W$ and the dynamical variables (individual preferences). This consensual and dynamic network, which can be seen as a kind of non supervised learning algorithm, is able to modify some individual preference variables through the process time. In conclusion, this approach models a dynamic network that minimises the cost to reach agreement amongst the experts. Subsequently, Fedrizzi et al. [18] extended their dynamical approach to the case of having uncertain preferences modelled as triangular fuzzy numbers. Recently, a new version of this dynamic approach that implements randomness in the modelling of the individual process iterations leading to singular (different) consensus process paths, with the aim to better capture the nature of some real world consensus reaching processes, was presented in [32]. The imprecision of the different consensus results is taken as the input of other simple process to obtain a collective consensus result from the distribution of the singular ones. Specifically, they introduce the randomness into the resistance component of the cost function by using a parameter as a standard normally distributed random component (with mean zero and variance one) divided by the number of iterations. In such a way, the effect of changing the step size is created and random experts are allowed to back-up in the discussion process.

A second point of view about improving the process convergence by modifying the experts' preferences at each iteration is given by Herrera-Viedma et al. [1, 2, 22, 39]. In this case, the authors do not minimise the cost function as described before, they focus on maximizing the experts' knowledge on the current discussion process state. In such a way, not only the feedback mechanism is extended to produce the most complete recommendations to the experts but also some algorithms to deal with ignorance or missing preference values based on experts' consistency are developed. These authors design simple but understandable and easy to use rules to support experts in changing their opinions. Indeed, the rules devised by these authors establishing the direction of change that the experts should follow to increase the group consensus. This is accomplished by computing consensus degrees to measure how far away are any two individual experts' preferences, and proximity measures to quantify how far each each individual expert's preferences are from the group's preferences, this last obtained by fusing the individual experts' preferences. These measures are computed at the three levels of a preference relation (pair of alternatives, alternative, and relation levels), which is exploited to identify the experts of the group whose contribute to consensus is insufficient, and in which alternatives and pair of alternatives preference values such contribution to consensus is specifically insufficient (identification rules) and to establish the direction of change of preference values (direction rules) to increase the group consensus.

A different approach to the two described above has been proposed by Parreiras et al. in $[35,36]$. In [36], the authors combine the different points of view on how to proceed making group decisions, with the moderator taking part in the discussion process by inviting any expert to update his/her preferences but also by adjusting the weight allocated to each expert's opinion that maximise the group soft consensus index. In [35], they propose a 
dynamical consensus scheme that, in addition to the soft consensus index, makes use of a comparability (similarity or credibility) and a concordance indices to control the flow of information during the negotiation process. The concordance index is used to choose an expert (usually the most discordant one) to be requested to explain to the other experts or to review his/her preferences, while the comparability index is used to select the subset of experts that had some problems in expressing their opinions so that they are supported by the most assertive experts in constructing their own preferences. By supporting an intense flow of information among the group, it is demonstrated that the process of sharing important information or knowledge is upgraded (at a risk of a longer negotiation process) and, therefore, the quality of the collective solution is also improved. Eventually, if one of the following circumstances is satisfied, the negotiation process is finished: (1) the consensus level has reached the established threshold; (2) the number of discussion round has reached the established threshold; (3) after a specific number of rounds, the same expert remains as the most discordant one and the moderator cannot help him/her to change his/her own opinion any more; (4) the previously established negotiation time has finished.

Finally, Cao et al. [8] presented a monitor based approach to deal with the following dynamic aspects of the consensus process: convergence rates, measurements delays and asynchronous events. To do so, they used properties of composition of directed graphs together with results from the theory of non homogeneous Markov chains to derive the worst case convergence rates for the headings of a group of experts performing a consensus process. This approach also uses graph theoretic construction to solve consensus processes with measurement delays, asynchronous events or a group leader. In [7], Cao et al. presented a graphical approach to the same problem. In order to establish the graph of a stochastic matrix, they define the concepts of rooted, strongly rooted and neighbour shared. Recently, following the monitoring idea, Palomares et al. [34] presented a new graphical monitoring tool of preference evolution. Furthermore, Wu et al. [44, 45] presented some similar approaches based on visual information to improve the consensus reaching process.

\subsubsection{Fast Convergence}

Usually, in real time situations, the quality of the collective decision is as important as the time it takes to make such decision. This is the case of decision making in geographic context, in business, in navigation applications or in natural resources management. Thus, a GDM process should be rapid so that the experts can yield and process information quickly. Furthermore, there are decision situations where some of the decision process data can be modified over time. Therefore, some researchers have focused on implementing previous theoretical consensus models via new information technologies such as web sites, smartphone apps or even implementing the GDM model as a web service [1, 24, 39, 40, 50]. They claim that if the experts have the opportunity to follow the negotiation process in real time, the decisions will improve. In [39], the concept of Mobile Decision Support System (MDSS) was proposed with the incorporation of mobile technologies in classical Decision Support Systems. It allows experts to have timely and updated information, anytime and anywhere, leading to an agile decision process by simulating real face to face negotiation meetings.

Additionally, shortening the negotiation time will limit the the number of changes in the parameters of the problem.

Table 1 summarises the advantages and drawbacks of the most important approaches 
analysed at this level.

\begin{tabular}{|c|c|c|}
\hline & Advantages & Drawbacks \\
\hline $\begin{array}{l}\text { Change of } \\
\text { Preferences }\end{array}$ & $\begin{array}{l}\text { Models support multi-stage pro- } \\
\text { cesses to modify experts' prefer- } \\
\text { ences if the consensus level is not } \\
\text { satisfactory. } \\
\text { Automatic feedback mechanism } \\
\text { improves the convergence of the } \\
\text { consensus process. }\end{array}$ & $\begin{array}{l}\text { The rest of the parameters of the } \\
\text { problem are assumed static. } \\
\text { Models not able to address environ- } \\
\text { mental changes. }\end{array}$ \\
\hline $\begin{array}{l}\text { Fast Con- } \\
\text { vergence }\end{array}$ & $\begin{array}{l}\text { Models speed up the information } \\
\text { flow by using new technologies. } \\
\text { Chances of changing the problem } \\
\text { parameters is reduced. }\end{array}$ & $\begin{array}{l}\text { Models not able to address be- } \\
\text { havioural changes. }\end{array}$ \\
\hline
\end{tabular}

Table 1: Classical Dynamic Consensus Approaches.

\subsection{Time Modelling Consensus Approaches}

Some researchers have considered time as the most important element for modelling dynamism, and as such the following two research trends in consensus reaching processed have emerged recently: (1) opinion dynamic modelling and (2) multi-period decision making.

\subsubsection{Opinion dynamic modelling}

Hegselmann et al. [21] proposed a time dependent approach to deal with changes during the negotiation process of previous classical models and social influence networks and opinion change. This new approached is based on the bounded confidence concept, where an expert is able to form his/her opinion by sharing other agents' opinions. Specifically, the opinion change evolution at time $(t+1)$ is modelled as the weighted arithmetic mean of opinions in the previous time stages $(t),(t-1), \ldots,(t-n)$. The crucial point here is that each expert associates weights to the opinion of others in order to establish the bounded confidence. Only in the case of constant weights and enough confidence among experts the consensus can be modelled with a classical approach. Therefore, the dynamism is in the computing of the opinion evolution taking into account the changes of others' opinion weights. In this way, experts can adjust their confidence in other opinions and their preferences are automatically updated by the system. This model is also known as the Hegselmann-Krause (HK) model model. On the other hand Deffuant et al. [11] presented the Deffuant-Weisbuch (DW) model to mix beliefs among interaction agents that has also become a classical bounded confidence model. Both models use the concept of bounded confidences, but differ in how many other experts each expert can interact with at a certain time.

Based on the DW model and the HK model, scholars have developed some interesting extensions, such as the linguistic version of the HK model developed by Dong et al. [14]. Another worth mentioned approach was proposed by Li et al. [28], where a new automated method based on a basic unit-interval monotonic (BUM) function was proposed to compute the time weight. 


\subsubsection{Multi-period group decision making}

In 2008, Xu [46] proposed a multi-period decision making concept by which experts may give different views about the same set of alternatives over a time period due to changes in the decision context. Therefore, it was considered necessary to incorporate in the consensus computation not just the latest preferences of each expert but also their previous expressed ones during the whole negotiation time period. This was modelled via a fusion of all the preferences provided by the an expert, the output of which was considered by this scholar the associated 'timeless preference of each expert'. The fusion operator used was termed the the dynamic weighted averaging (DWA) operator, which incorporates the variable time $(t)$, with three variants: arithmetic series, geometric series, and normal distribution based DWA operators. Also, the Uncertain based DWA operator (UDWA) was proposed to aggregate interval valued arguments. This strategy has been taken forward to deal with intuitionistic fuzzy information, linguistic information [47-49], and hesitant preferences [37], respectively.

Recently, Gupta et al. [20] proposed a different approach to the multi-period decision making problem, which is based the concept of expert's net preferences $(N P)$ of alternatives in pairwise form after accounting his/her judgements in different time intervals over a period, which are subsequently transformed a set of sequences. These sequences are called maximum sequences and represent the group's final alternatives rankings. Figure 2 shows this approach.

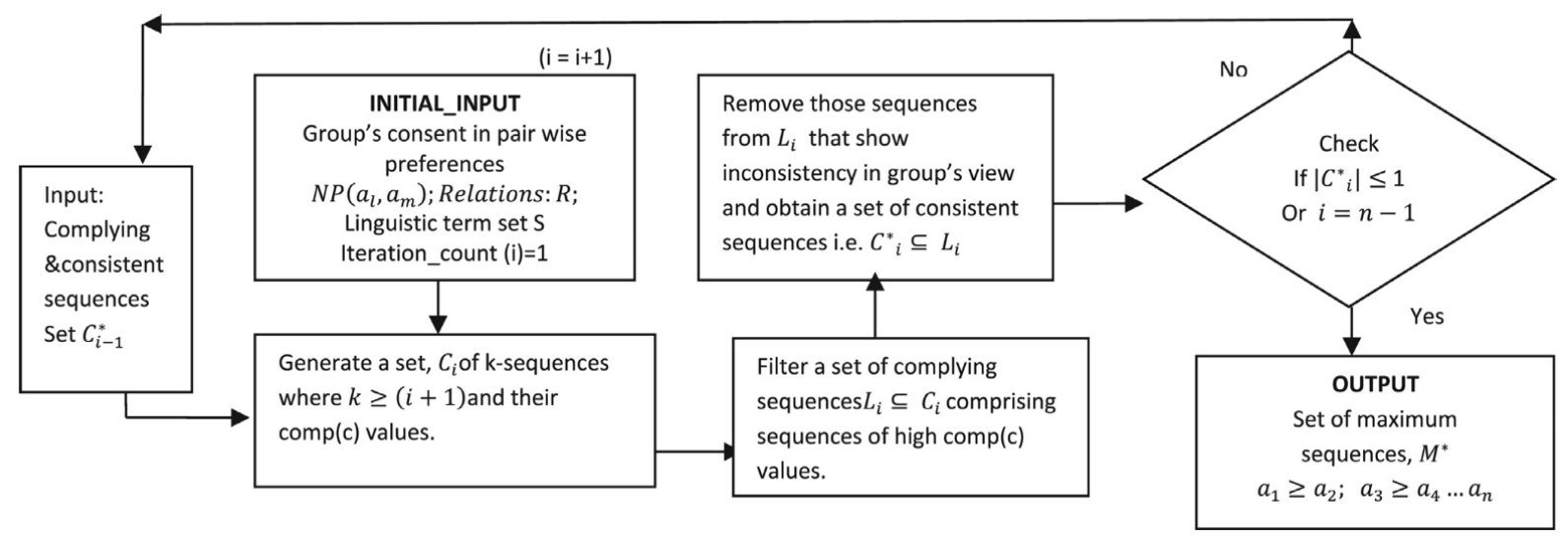

Figure 2: Ranked list algorithm process flow [20]

Table 2 summarises the advantages and drawbacks of the most important approaches analysed at this level.

\subsection{Dynamic Environment in Consensus Approaches}

In this section we analyse some consensus approaches that model the problem environment as dynamic. These are classed into four different groups: 1) Dynamic alternatives management, 2) Dynamic experts management, 3) Dynamic experts' importance management, and 4) Dynamic criteria management

\subsubsection{Dynamic set of alternatives}

As mentioned before, the set of alternatives can change during the decision making process, with some of the alternatives' feasibility or availability being affected (changed) 


\begin{tabular}{|l|l|l|}
\cline { 2 - 3 } \multicolumn{1}{c|}{} & \multicolumn{1}{c|}{ Advantages } & \multicolumn{1}{c|}{ Drawbacks } \\
\hline $\begin{array}{l}\text { Opinion } \\
\text { Dynamics }\end{array}$ & $\begin{array}{l}\text { Models are able to compute a } \\
\text { sound prediction of experts pref- } \\
\text { erences evolution. } \\
\text { Decrease time of waiting for the } \\
\text { real opinion changes. }\end{array}$ & $\begin{array}{l}\text { Prediction of other problem pa- } \\
\text { rameters evolution is not possible. } \\
\text { The computed prediction could } \\
\text { fail in some cases. }\end{array}$ \\
\hline $\begin{array}{l}\text { Multi-Period } \\
\text { GDM }\end{array}$ & $\begin{array}{l}\text { These approaches consider a prob- } \\
\text { lem snapshot in time. } \\
\text { Different opinions of the same ex- } \\
\text { pert at different moments are ag- } \\
\text { gregated to obtain an accepted } \\
\text { overall opinion. }\end{array}$ & $\begin{array}{l}\text { She opinions are the only change- } \\
\text { able parameter. } \\
\text { overall opinion should be the lat- } \\
\text { est one instead of the aggregation } \\
\text { of all the intermediate ones. }\end{array}$ \\
\hline
\end{tabular}

Table 2: Time Modelling Consensus Approaches.

during the process time. For example, in medical diagnosis scenarios where the treatments to recommend to the patient are the alternatives to select from. In such a case, medical doctors (experts) should take into account any variation of the patient state in the previous few hours, as the patient health could have improved or deteriorated (presenting new health symptoms) due to the prescribed medication. Another typical example of this situation is e-commerce or e-business decision scenarios, with the different products or services available to purchase form the set of alternatives to consider. In this particular case, it is easy to see that, while the negotiation details (prices, quantity, etc...) is underway, the product/services availability could change. This issue of dynamic alternatives in decision making has been addressed by Pérez et al. in [39-41], where a method to remove the 'worst' alternatives and insert new ones into the discussion process was proposed. The proposed procedure differentiates the two cases: (1) remove old worst alternatives, and (2) insert new good alternatives.

(1) The first case deals with situations where some alternatives are not available any longer because they have been discarded by the experts of due to external factors to the group of experts. From the set of alternatives, candidates alternatives for removing are those ranked below a threshold value in terms of their associated dominance degree $(Q G D D)$ (see Figure 3a).

(2) The second case is managed by the system, with a corresponding module informing when a new 'good' alternative appears in the decision context to replace the one with lowest $Q G D D$ (see Figure 3b).

Finally, in both cases, experts have to express their agreement or disagreement with the changes proposed by the system. If most of the experts approves the changes, the system makes the necessary adjustments in the set of alternatives ready for the next consensus stage. In order to correctly integrate this new tool into the consensus reaching process, the feedback 


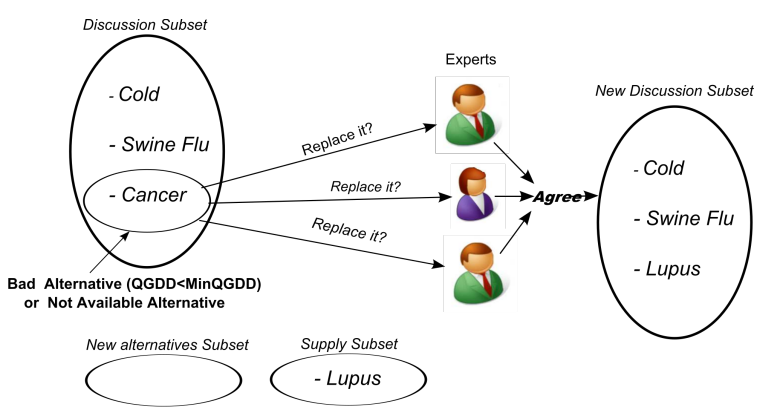

(a) Case 1

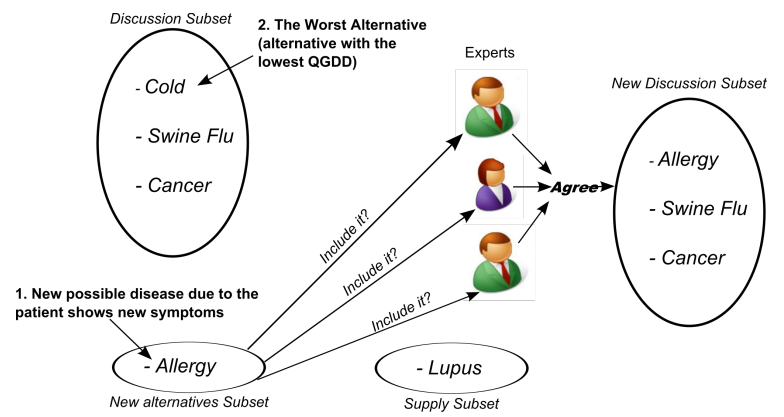

(b) Case 2

Figure 3: Dynamic alternatives management [39]

mechanism is also updated to disregard as unnecessary any recommendation that refers to any of the discarded alternatives.

\subsubsection{Dynamic set of experts}

Commonly, the number of people involved in a group decisions process can change with some people leaving the process with others replacing them or not. Moreover, when a large amount of individuals is involved in a negotiation process, the decision task becomes harder, although this is possible now with new (mobile, internet enabled) technologies viable for the decision making process management. For example, in social media or web communities environments new expert could be incorporated to the ongoing discussion process at anytime, and some of the current experts leaving the process as well. Alternatively, a big group of people could be reduced in order to simplify communications and to facilitate the consensus reaching process. Some scholars have focused their research efforts on modelling these aspects of the negotiation processes.

Ballester et al. in [3] presented a recursive procedure to select competent subgroups of experts just taking into account their own opinions about the whole group. However, the most important dynamic approach to deal with these decision problems is by Alonso et al. in [2], where they designed a sophisticated consensus reaching model that incorporates a delegation scheme to manage large and dynamic groups of experts in which they may choose to continue the discussion process or delegate other experts, normally with similar preferences (see Figure 4). The introduction of a delegation mechanism can solve the problem of sporadic contributions that appears in web communities and social networks and reduce the amount of individual opinions related to the decision problem. Some details on the proposed delegation scheme are presented in the following:

1. At each discussion round an expert $e^{h}$ can freely delegated to other one $t^{h}$ and leave the decision making process or revoke a delegation previously made. In that case, he/she will not be requested to update his/her preferences anymore.

2. The delegation mechanism can be interpreted as a trust network and, consequently, be modelled as a directed graph. In such a way, transitivity conditions can be applied. Moreover, in order to avoid cycles, the system will produce alerts and prevent delegate to an expert that had previously (directly or indirectly) delegated to that expert. 


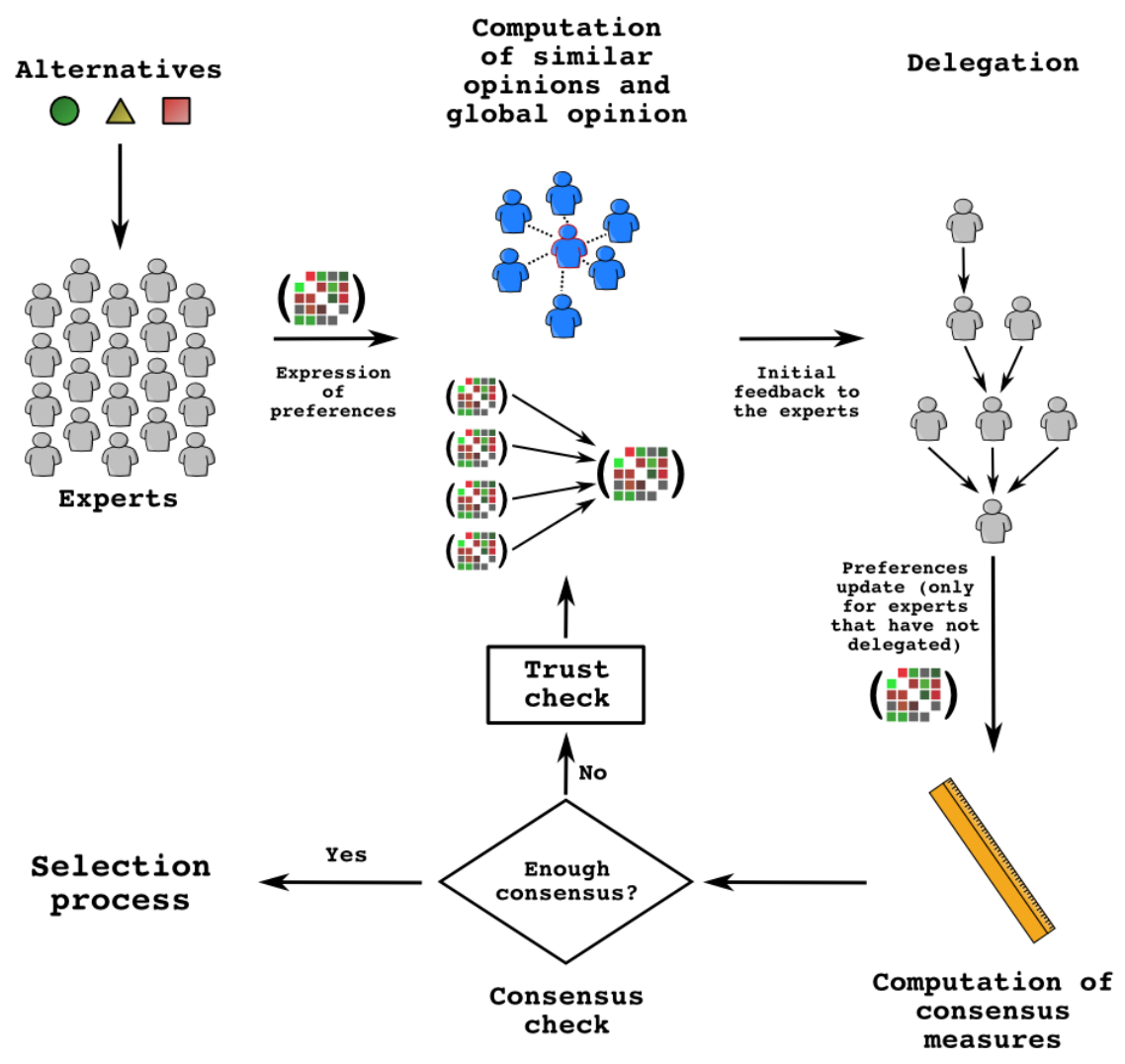

Figure 4: Delegation scheme in the consensus model of [2]

3. After the delegation stipulated time, the system will summarise all the proposed delegations by using trust weights vector $\tau^{h}$, which are related to how many times an expert has been delegated.

4. A trust checking mechanism is incorporated to avoid malicious experts. In such a way, if an expert with a trust weight $\tau^{h}>0$ changes his/her preferences drastically in the next round of the consensus process, those experts that delegated to him/her are informed and allowed to cancel these delegations if they consider that their preferences are no longer appropriately represented.

\subsubsection{Dynamic experts' importance}

A key issue in GDM is the importance or weight associated to experts [13]. Saaty in [43] propose a process to determine experts' weights by taking into account prior information on the experts using a hierarchy of criteria such as experience, expertise, persuasive abilities, previous performance and effort on the problem to solve; while Ramanathan et al. [42] proposed an eigenvector based method to compute the weight vector by using interpersonal comparison among experts. However, Forman et al. [19] noted that sometimes it is not easy to find a knowledgeable person to provide assessments based on Saaty's hierarchy approach or he/she may not have enough knowledge to rank the experts' importance. Therefore, without prior information there us a need to have an objective way to determine the weights associated to the experts. Once, experts' weights are determined, a weighted average of 


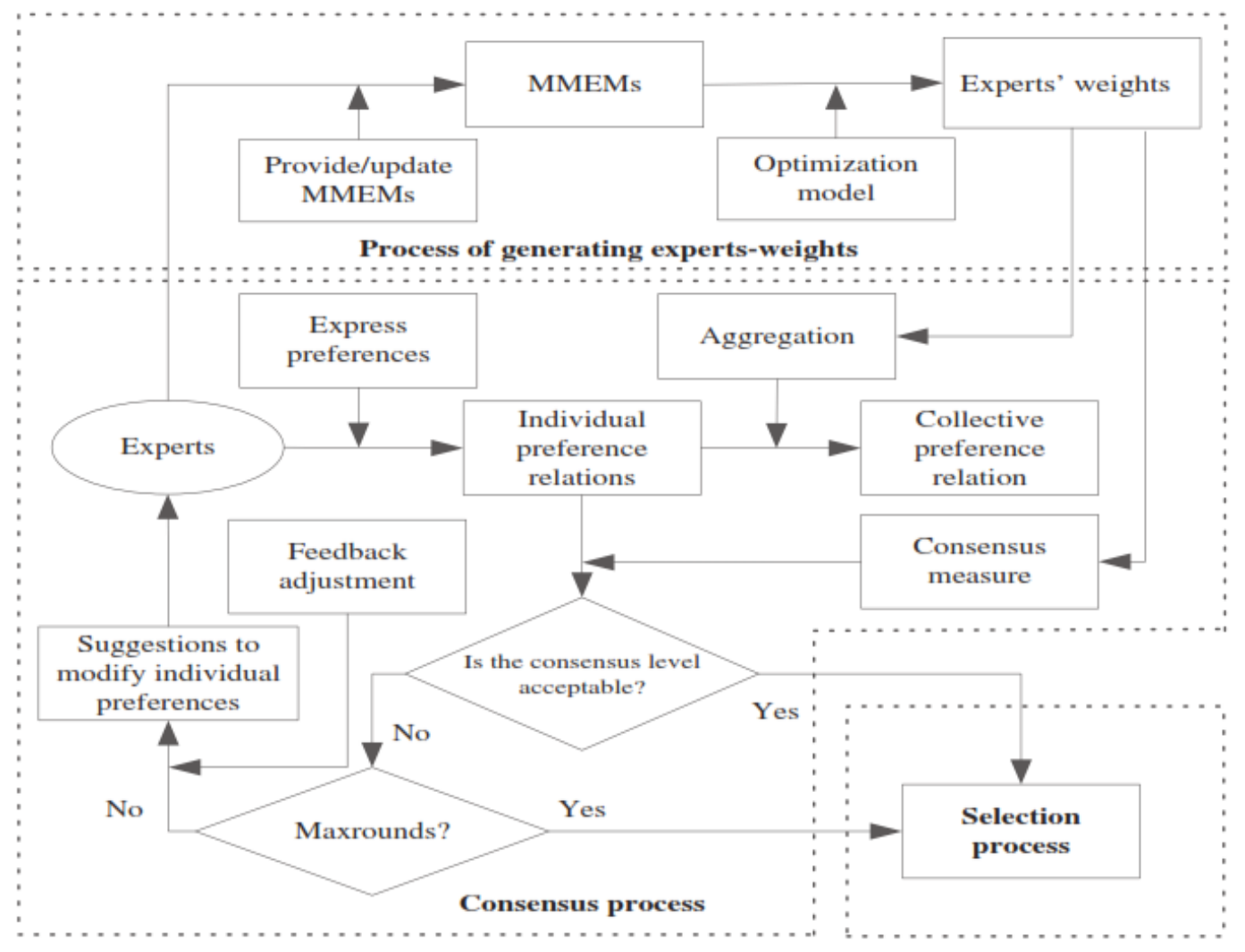

Figure 5: Non-cooperative behaviours in GDM [16]

their opinions or preferences is computed to derive the collective/group opinion or preference. Experts' weights are considered static, constant during the whole the decision process. However, it is obvious that in a dynamic environment, experts' importance may vary during the decision process. In recent years, some dynamic consensus models that incorporate a dynamic way of determine experts' weights have been developed.

- Dong et al. [13] proposed to determine the weights based on experts' opinion transition probabilities. They state that the differences in the proximity between experts reveal the possibilities of opinion transitions. This opinion transition process can be modelled by using a finite state space Markov chain. Considering the limiting distribution of the chain, the authors calculated the power or weight distribution of experts in a group. Eventually, Dong et al. [16] proposed another approach to integrate experts' weights dynamically generated into the consensus process. In this proposal, the experts provide not only mutual evaluation information for the other experts, but also preference information about alternatives. This mutual evaluation is represented by using multi-attribute mutual evaluation matrices (MMEMs). The authors proposed an optimization-based way to obtain the experts' weights from the MMEMs (see Figure 5).

- Li et al. [29] proposed a method for adjusting weights by using an analytic method; which was extended in [27] via the application of a Borda function and linguistic quantifier to construct the variable weight state vector. 


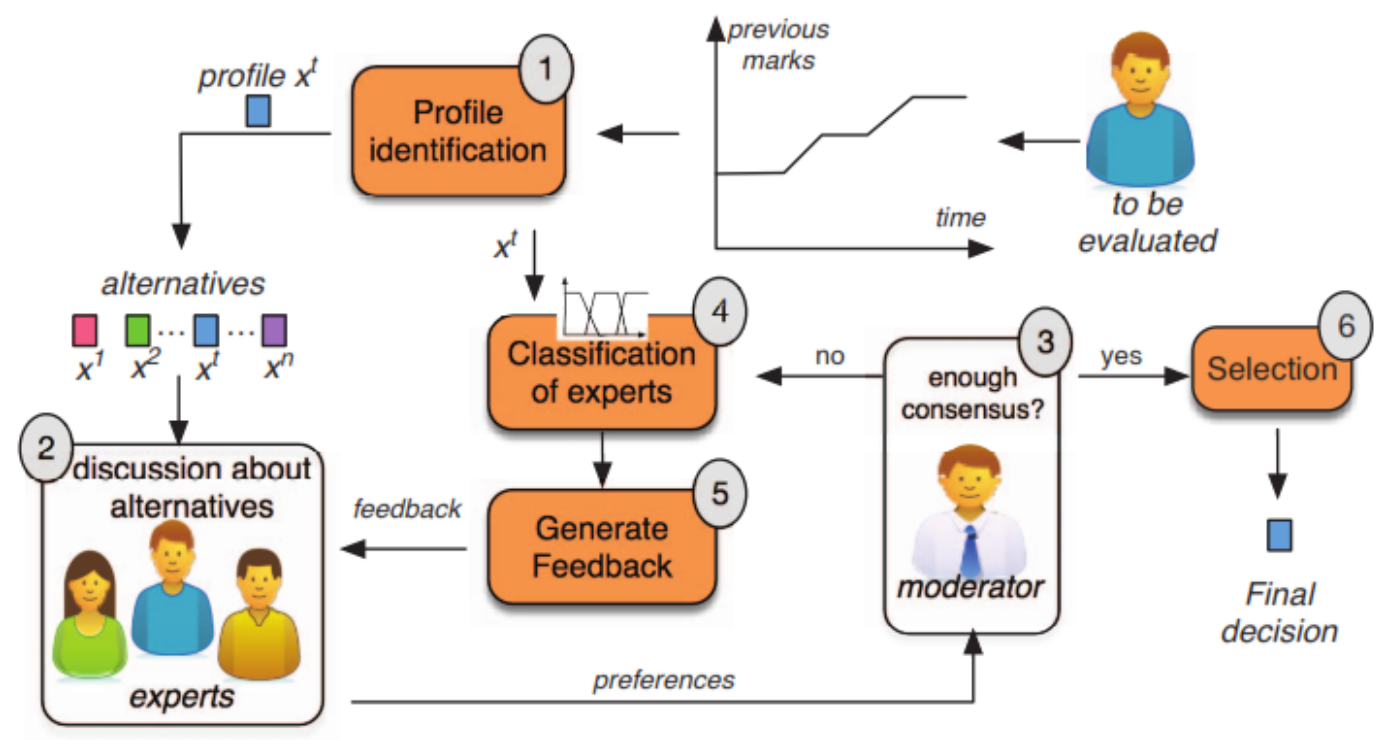

Figure 6: Experts profile in GDM situations [10]

- D'Aniello et al. [10] proposed a consensus approach with variable importance of experts via the incorporation of the concept of expert's profile, which contains useful information about the experts that is subsequently used compute dynamically the experts' weights depending on each situation (see Figure 6).

\subsubsection{Dynamic set of criteria}

Frequently, in GDM problems, it is necessary to take into account different criteria to assess the alternatives. For example, an alternative could be the best one based on a quality criterion, but the worst one based on a price criterion. Thus, the evaluation by using more than one criterion is necessary to derive usually the overall best alternative. Multi-criteria decision making (MCDM) refers to a process to derive the best alternative from a feasible set which are assessed against multiple and sometimes conflictive criteria. Most of the current multi criteria consensus models consider the criteria set as a fixed set or static parameters of the consensus process; although recently, new approaches have been developed that allow the set of criteria to be modelled as dynamic parameters of the consensus process.

- Lourenzutti et al. in [31] proposed a generalized TOPSIS (Technique for Order Preference by Similarity to Ideal Solution) in a dynamic environment. They introduced the GMo-RTOPSIS (the Modular TOPSIS in random environments for Group decision making) that treats each expert independently and with complete freedom to express their opinions. Additionally, the experts are not forced to agree with an specific criteria set, and as such experts can consider different criteria sets and different underlying factors, weight vectors or mappings. The proposed method considers each criterion of each expert as a separate module, and random variables are used to deal with underlying factors. Summarising, this approach is able to deal with real decision situations where the unpredictable environment can affect the performance of the alternatives. 


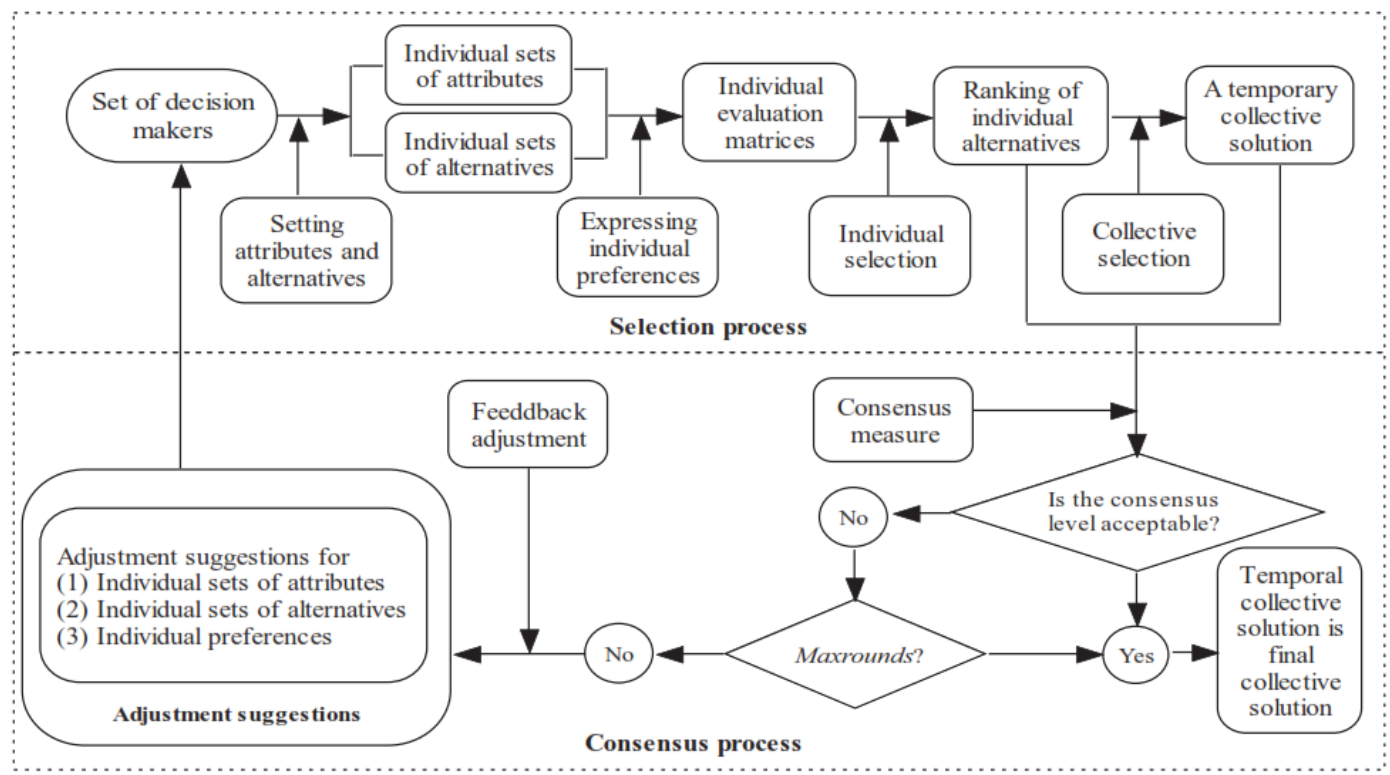

Figure 7: The resolution framework for complex and dynamic MCGDM [15]

- Dong et al. [15] have also proposed a consensus reaching model for dynamic and complex MCGDM (Multi Criteria Group Decision Making) problem. In this approach, each expert has an individual set of attributes or criteria and an individual set of alternatives, which undergo some dynamic changes through the consensus reaching process. The main feature of this proposal is that it is not necessary to reach an agreement among the experts; its aim or challenge being to be able to find one alternative authorised by all, or at least, most of the experts. This method is graphically depicted in Figure 7.

Tables 3 summarises the advantages and drawbacks of the most important approaches analysed at this level.

\subsection{Adaptive Consensus Approaches}

The basis of adaptive consensus models is a refinement or sophistication of the process to allow the agreement increasing at the same time that the quality and amount of advice produced by the system are improved and reduced, respectively [6]. In other words, the generated recommendations are adaptively selected by taking into account some of the dynamic parameters of the problem. In the following, the main adaptive consensus approaches and their results are described.

\subsubsection{Feedback mechanism adaptation to the collective consensus level}

One of the first adaptive consensus models was presented by Mata et al. [33], where the amount of generated recommendations at each discussion iteration was adapted to the current consensus degree. Indeed, the level of consensus affects the amount of changes required by a group of experts to facilitate the reaching of agreement. When the consensus level low a higher amount of advice to support experts to close their preferences is required than 


\begin{tabular}{|c|c|c|}
\hline & Advantages & Drawbacks \\
\hline $\begin{array}{l}\text { Dynamic Set of } \\
\text { Alternatives }\end{array}$ & $\begin{array}{l}\text { The set of alternatives is dynamic } \\
\text { and can vary through the discus- } \\
\text { sion process time. } \\
\text { Large sets of alternatives could be } \\
\text { taken into account. }\end{array}$ & $\begin{array}{l}\text { The four different approaches } \\
\text { are applied independently. } \\
\text { Thus, their combined appli- } \\
\text { cation should be preferred in } \\
\text { order to deal with real world } \\
\text { decision problems. They just } \\
\text { solve isolated parts of the whole } \\
\text { problem. }\end{array}$ \\
\hline $\begin{array}{l}\text { Dynamic Set of } \\
\text { Experts }\end{array}$ & $\begin{array}{l}\text { The set of experts is dynamic and } \\
\text { experts involved in the consensus } \\
\text { process could vary through the } \\
\text { discussion process. } \\
\text { Large sets of experts (e.g. web } \\
\text { communities) could help to take } \\
\text { the best decision. } \\
\text { The proposed delegation scheme } \\
\text { and the trust network allow to } \\
\text { put in place group representa- } \\
\text { tives. }\end{array}$ & $\begin{array}{l}\text { The four different approaches' } \\
\text { behaviour is always the same, } \\
\text { regardless of the environment } \\
\text { changes }\end{array}$ \\
\hline $\begin{array}{l}\text { Dynamic Ex- } \\
\text { perts' Impor- } \\
\text { tance }\end{array}$ & $\begin{array}{l}\text { Experts' importance is dynamic } \\
\text { and the weight of each expert at } \\
\text { the preferences aggregation could } \\
\text { vary through the discussion pro- } \\
\text { cess. } \\
\text { Different ways to determine ex- } \\
\text { perts' weight dynamically have } \\
\text { been proposed. } \\
\text { Non-cooperative behaviours have } \\
\text { been taken into account. }\end{array}$ & \\
\hline $\begin{array}{l}\text { Dynamic Set of } \\
\text { Criteria }\end{array}$ & $\begin{array}{l}\text { The criteria set is dynamic and } \\
\text { the criteria to use in assessing } \\
\text { the set of alternatives could vary } \\
\text { through the discussion process. } \\
\text { Each expert is allowed to have } \\
\text { his/her own criteria set. }\end{array}$ & \\
\hline
\end{tabular}

Table 3: Dynamic Environment in Consensus Approaches 


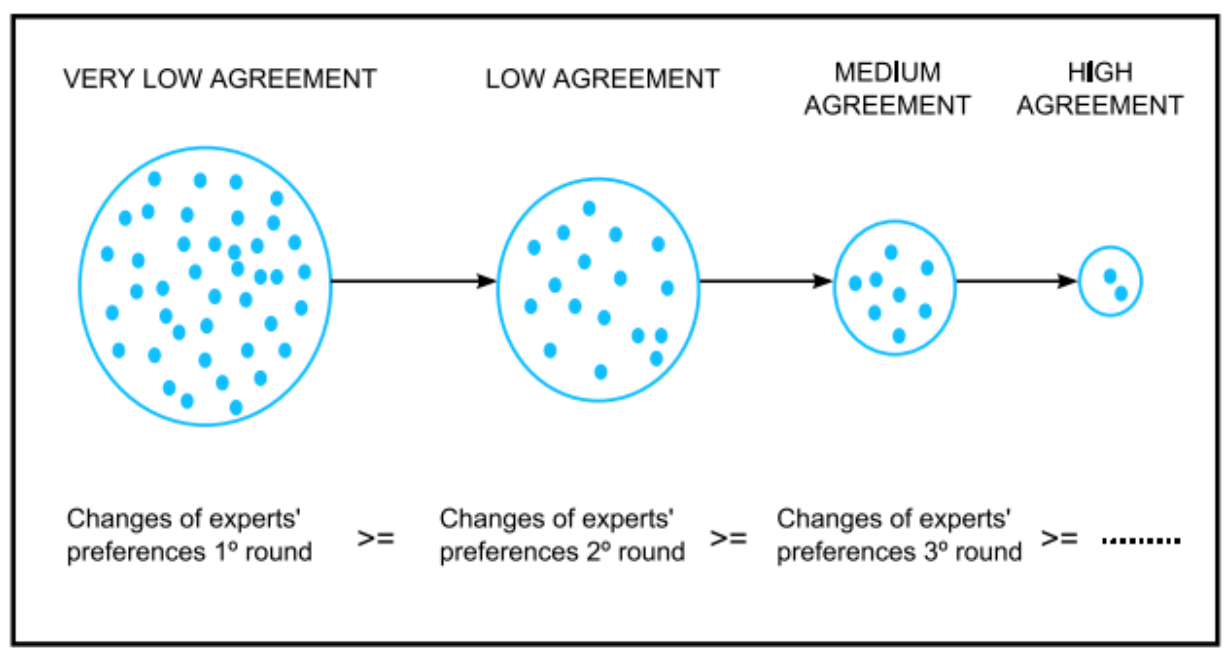

Figure 8: Reduction of the amount of advice into the discussion process [33]

when the consensus level is high . Specifically, these scholars propose a dynamic procedure of searching preferences, which they term PSp, to change for improving the agreement level based on the current consensus level achieved at the specific consensus round. As mentioned before, as the consensus round increases there will be an increase of the consensus level and therefore this will lead to a decrease of the amount of recommendations of preference changes the experts will receive. The PSp dynamic strategy implemented is based on the classification of the agreement in four categories: very low; low; medium; and high. Figure 8 illustrates the described PSp dynamic strategy.

\subsubsection{Feedback mechanism adaptation to the experts' importance}

A second adaptive consensus model was presented by Pérez et al. [38]. This model is characterised by a novel feedback mechanism that adapts the amount of recommendations to the experts' weight or importance level. Experts with high expertise level normally need less advice compared with others with lower expertise. Thus, experts are classified into three different classes: (1) high-important experts, $E_{h i g h},(2)$ medium-important experts, $E_{m e d}$, and (3) low-important experts, $E_{\text {low }}$. It is worth noting that one of these categories could be empty, and therefore there could be situations where there are three subclasses of experts based on their level of expertise, or two subclasses to just one subclass. These three possible subclasses of expertise lead to three different strategies for selecting how many preferences values to change in a consensus round to increase the group consensus level: (i) advising lowimportant experts, (ii) advising medium-important experts, and (iii) advising high-important experts (see Figure 9). The higher the expertise level, the less changes will be recommended by the feedback process. Furthermore, as covered in a previous section, the experts' weight can be changed dynamically, which makes this consensus reaching model to be an adaptive and dynamic consensus reaching model.

\subsubsection{Feedback mechanism adaptation to the individual consensus indeces}

Recently, a novel peer to peer dynamic adaptive consensus reaching model has been proposed by Dong et al. [13]. In comparison with centricity oriented methods, which use the 


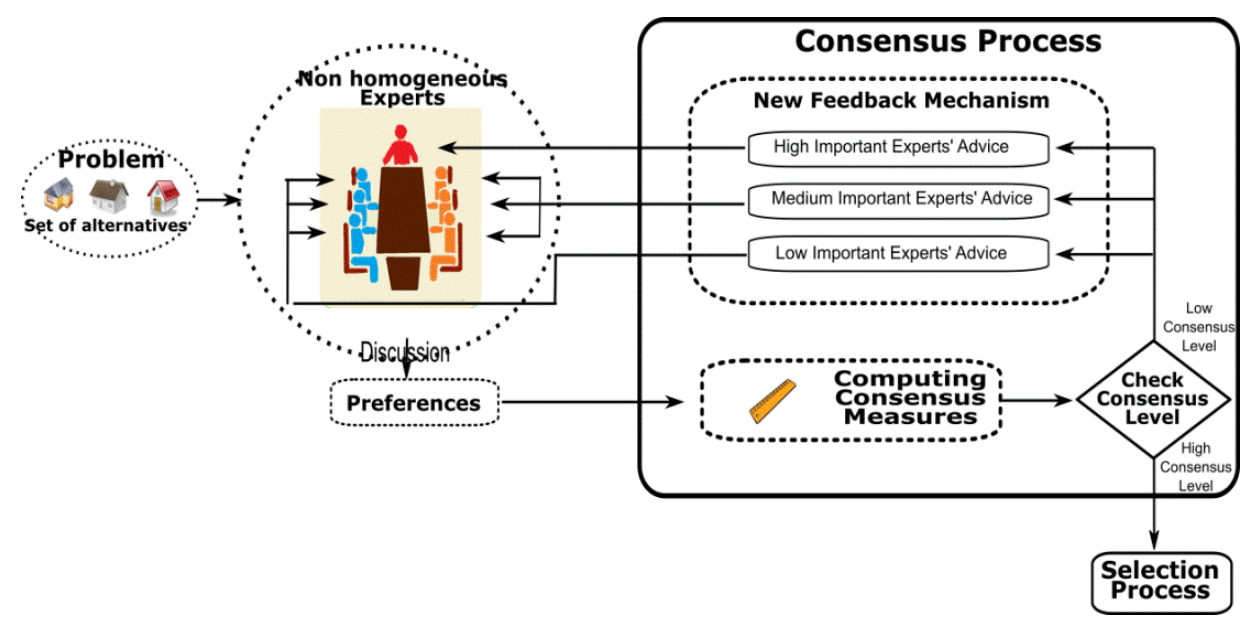

Figure 9: Consensus reaching process with non-homogeneous experts [38]

aggregated group opinion as the reference point in the consensus reaching process [33, 38], this new adaptive proposal compares the information from one expert to another one. This peer to peer comparison strategy reduces the computation cost and biases in the aggregation process. Experts' importance degrees are determined using a Markov chain allocating method based on the opinion transition probability. The Markov chain models the probabilities when it is expected that differences in the proximity among experts reveal the possibilities of changes in their preference. The model assumes it is more probable for an expert to change his/her opinion closer to those of similar experts than to those of dissimilar experts. An issue of this approach resides in the slow or even ineffective convergence of the consensus process when small changes of preferences happen. The challenge then becomes on how to assist experts in making bigger changes on their preferences. However, the authors noticed that if non compatible experts, i.e. experts whose individual opinions are far away, are selected and their preferences are changed to be closer then a strong increase of the consensus level will be achieved. This principle is used by the authors to proposed a new convergent and effective algorithm that implements a feedback mechanism that in each round provides recommendations to just only those pairs of experts with maximum Individual Consensus Index (ICI) in contrast to previous approaches based on a set of rules that apply to all experts with ICI below the acceptable threshold [33, 38]. Therefore, as the ICI changes dynamically, in response to the experts updated preferences, and the recommendations' recipients are selected depending on this index, the authors defined the approach as dynamic and adaptive (see Figure 10).

Table 4 summarises the advantages and drawbacks of the most important approaches analysed at this level.

\section{Discussion and future trends}

Many dynamic consensus methodological approaches have appeared in recent years, which have been reviewed and analysed in the present paper in terms of their associated advantages and drawbacks. The main findings of this present review are: 


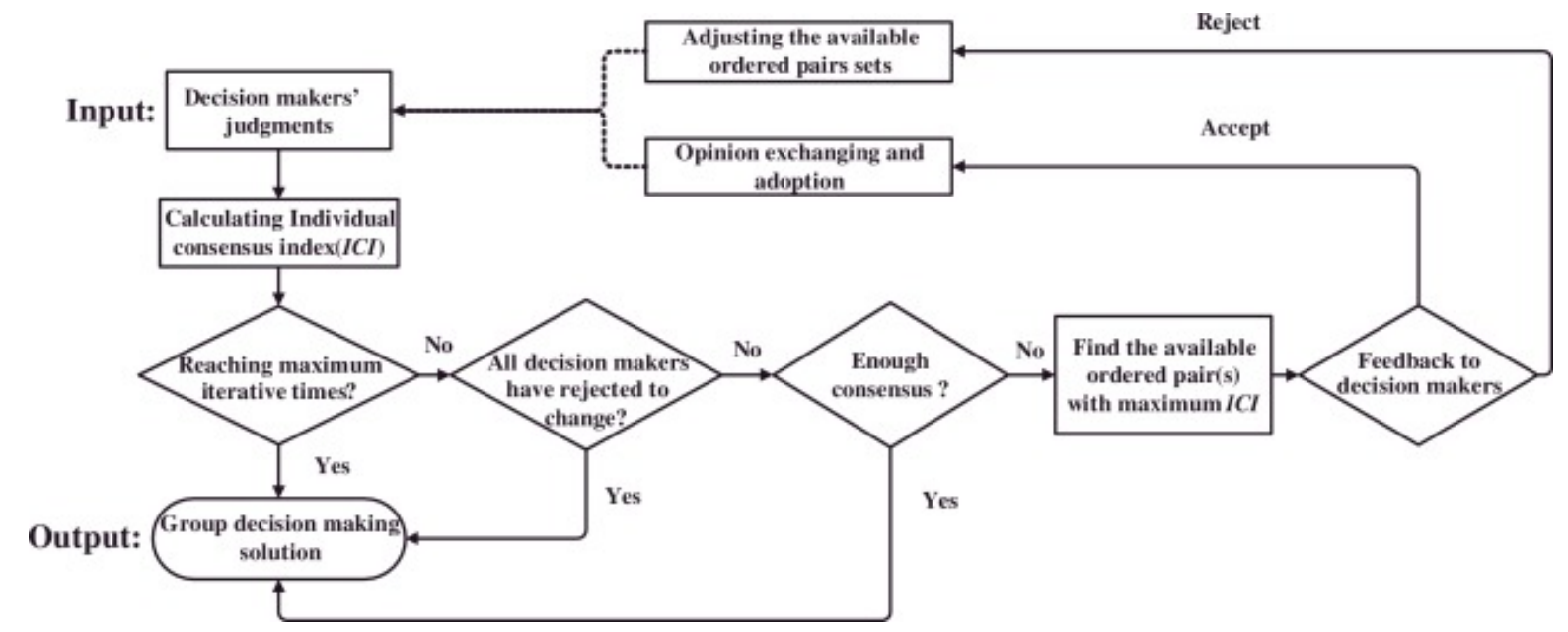

Figure 10: Adapting the feedback mechanism to the individual consensus indexes [13]

\begin{tabular}{|c|c|c|}
\hline & Advantages & Drawbacks \\
\hline Collective CL & $\begin{array}{l}\text { The behaviour of the model is dif- } \\
\text { ferent depending on the current } \\
\text { collective consensus level. } \\
\text { If the consensus level is low, the } \\
\text { amount of recommendations is } \\
\text { bigger than otherwise. }\end{array}$ & $\begin{array}{l}\text { The behaviour of the model is } \\
\text { modified but it does not allow } \\
\text { changes of the problem parame- } \\
\text { ters. }\end{array}$ \\
\hline $\begin{array}{l}\text { Experts' } \\
\text { Importance }\end{array}$ & $\begin{array}{l}\text { The behaviour of the model is dif- } \\
\text { ferent depending on the expert } \\
\text { importance. } \\
\text { The higher the importance of an } \\
\text { expert the less recommendations } \\
\text { receives. }\end{array}$ & $\begin{array}{l}\text { Experts' importances remain } \\
\text { fixed through the discussion } \\
\text { time. }\end{array}$ \\
\hline Individual CI & $\begin{array}{l}\text { The behaviour of the model is dif- } \\
\text { ferent depending on the current } \\
\text { individual consensus index. }\end{array}$ & $\begin{array}{l}\text { The behaviour of the model is } \\
\text { modified but it does not allows } \\
\text { changes of the problem parame- } \\
\text { ters. }\end{array}$ \\
\hline
\end{tabular}

Table 4: Adaptive Consensus Approaches. 
1. The main approaches modelling dynamic change of preferences approaches lack dynamism in the rest of the problem elements. In these approaches, expert's preferences change and the consensus process converges but the other parameters remain fixed through the whole process.

2. There are dynamic approaches able to to speed up the information flow of the process, shortening the negotiation time and consequently, reducing the probability of change in the problem parameters. These approaches make possible to achieve a rapid convergence of the consensus process. However, if changes in the problem parameters happen, these models are not able to address them appropriately.

3. Opinion dynamic approaches are able to predict the evolution of the experts' preferences. Nevertheless, the possible changes or evolution of the others problem parameters are not taken into account; while multi-period GDM approaches focus on the opinions as the only changeable parameter.

4. The group of approaches that model the problem environment as a group of dynamic parameters (alternatives, experts, weights and criteria) focus on one environment parameter at a time, thus to completely address the dynamism of the consensus process all the problem parameters should be treated as dynamic. The main issue associated with the analysed approaches is that their behaviour is always the same, regardless of the environment changes.

5. Adaptive consensus approaches address the issue of the fixed/static behaviour mentioned above, as the corresponding models adapt their behaviour to the current problem situation in an efficient way.

6. Each one of the analysed approaches focused on a single dynamic aspect of the consensus problem. Thus, a combination of different models would be desirable in order to solve a real world consensus reaching problem.

The following future important research challenges in this field have been identified:

- Adaptive consensus modelling is still a hot topic that presents some good approaches but it could be improved in some aspects to address real world situations that have not been modelled yet. For example, it could be interesting to study and develop models that adapt the computation of the feedback advice to other dynamic parameters such as the experts' consistency or the expert's changing aversion.

- One key issue of the current consensus model is that experts acceptance of the advice received from the feedback mechanism to increase the consensus level and to achieve a convergent process is taken for granted. However, experts can decide not to implement the feedback recommendations and keep their original opinions/preferences. Therefore, there is a need to provide a framework that adds legitimacy to the feedback process to convince the experts to accept the provided advices. This can be achieved by implementing psychology concepts or persuasion principles in order to model the influence as an important part of the consensus reaching processes [9]. Persuasion principles are based on psychological studies about human behaviors. Therefore, if the consensus reaching processes incorporate some of these principles as a mean of influence, model with a higher power of persuasion to convince experts to be more flexible and reach the collective agreement will be possible. Several persuasion principles of interest are: 
- Returning a favour is usually a tendency among people.

- When people see other individuals doing something, they commonly do the same thing, even if they do not understand the reasons why.

- Authority figures normally impose some rules that others comply with no questions.

- Supply and demand law causes effect on people when they have to make decisions.

- When some people like an individual, they can be easily influenced by him/her.

- In any case, it is quite important to add some arguments to the provided opinions/preferences to support their correctness and help persuade others to follow you. Thus, dynamic and argumentative consensus models implementing argumentation mechanisms to the current consensus approaches are desirable. Credibility measures seems to be required for such approaches, which could also be modelled as an element of dynamic nature in the consensus process.

Summarising, we have identified two main research gaps that could be considered as new challenges to be addressed by the research community. The first one is the construction of new adaptive consensus models in order to make more realistic and efficient feedback mechanisms; while the second one refers to the need of new methods to deal with non cooperative experts where persuasion models and/or argumentation models might play a key role in modelling the consensus process.

\section{Conclusions}

In this paper, we have analysed in a comprehensive way some consensus approaches in which the model's parameters or properties have been specifically designed to change dynamically over the discussion time. To do that, we have proposed a taxonomy to classify the current dynamic consensus reaching models.

Classical dynamic consensus approaches are those that only admit changes on the experts' preferences, with the remaining parameter of the decision problem remaining fixed or static. The dynamisms can be introduced in the consensus process by incorporating a variable of time. Two different time modelling strategies have been identified: opinion dynamic approaches that aim at predicting the experts' preferences on a time state based on a set of previous time states; and multi period approaches that propose the aggregation of the different preferences at different time periods. A third group of reviewed approaches go a step further by modelling the decision environment as a dynamic one by allowing other problem parameters -set of alternatives; set experts set; experts' importance; or set of criteria- to change and not remain fixed throughout the consensus process. Finally, the last group of dynamic consensus approaches analysed have been the adaptive consensus approaches that incorporate implement feedback mechanisms that adapt at each consensus stage to the current collective consensus level, experts' importance, or individual consensus indexes. The most productive research avenues with their strengths and weaknesses have been analysed, and future research challenges have been pointed out. Table 5 summarises the most important analysed techniques, with corresponding their references, as per these four different methodologies 


\begin{tabular}{|c|c|c|}
\hline Methodologies & Techniques & References \\
\hline \multirow[t]{2}{*}{ Classical CA } & Change of Preferences & $\begin{array}{l}{[8,17,18,22,32,} \\
34-36,44,45]\end{array}$ \\
\hline & Fast Convergence & {$[1,24,39,40,50]$} \\
\hline \multirow[t]{2}{*}{ Time Modelling CA } & Opinion Dynamics & {$[11,14,21,28]$} \\
\hline & Multi-Period GDM & {$[20,37,46-49]$} \\
\hline \multirow[t]{4}{*}{ Dynamic Environment } & Dynamic Set of Alternatives & {$[39-41]$} \\
\hline & Dynamic Set of Experts & {$[2,3]$} \\
\hline & Dynamic Experts' Importance & $\begin{array}{l}{[10,13,16,27,} \\
29]\end{array}$ \\
\hline & Dynamic Set of Criteria & {$[15,31]$} \\
\hline \multirow[t]{3}{*}{ Adaptive Consensus Approaches } & Collective Consensus Level & {$[33]$} \\
\hline & Experts' Importance & {$[38]$} \\
\hline & Individual Consensus Indeces & {$[13]$} \\
\hline
\end{tabular}

Table 5: Taxonomy of methodologies and techniques for dynamic consensus approaches.

\section{Acknowledgments}

The authors would like to acknowledge the financial support from FEDER funds received from the Spanish Department for Economy and Competitiveness project (TIN2016-75850-R); the Chinese NSF grant (No. 71571124); and the University of Cadiz project (PR2016-026).

\section{References}

[1] S. Alonso, E. Herrera-Viedma, F. Chiclana, and F. Herrera. A web based consensus support system for group decision making problems and incomplete preferences. Information Sciences, 180(23):4477-4495, 2010.

[2] S. Alonso, I.J. Pérez, F.J. Cabrerizo, and E. Herrera-Viedma. A linguistic consensus model for web 2.0 communities. Applied Soft Computing, 13(1):149-157, 2013.

[3] M.A. Ballester and J.L. García-Lapresta. A recursive group decision-making procedure for choosing qualified individuals. International Journal of Intelligent Systems, 24(8):889-901, 2009.

[4] F.J. Cabrerizo, R. Al-Hmouz, A. Morfeq, A.S. Balamash, M.A. Martínez, and E. Herrera-Viedma. Soft consensus measures in group decision making using unbalanced fuzzy linguistic information. Soft Computing, 21(11):3037-3050, 2017.

[5] F.J. Cabrerizo, E. Herrera-Viedma, and W. Pedrycz. A method based on pso and granular computing of linguistic information to solve group decision making problems defined in heterogeneous contexts. European Journal of Operational Research, 230(3):624-633, 2013. 
[6] F.J. Cabrerizo, J.M. Moreno, I.J. Pérez, and E. Herrera-Viedma. Analyzing consensus approaches in fuzzy group decision making: advantages and drawbacks. Soft Computing, 14(5):451-463, 2010.

[7] M. Cao, A. Morse, and B. Anderson. Reaching a consensus in a dynamically changing environment: A graphical approach. SIAM Journal on Control and Optimization, 47(2):575-600, 2008.

[8] M. Cao, A. Morse, and B. Anderson. Reaching a consensus in a dynamically changing environment: convergence rates, measurement delays, and asynchronous events. SIAM Journal on Control and Optimization, 47(2):601-623, 2008.

[9] N. Capuano, F. Chiclana, H. Fujita, E. Herrera-Viedma, and V. Loia. Fuzzy group decision making with incomplete information guided by social influence. IEEE Trans. On Fuzzy Systems, Doi: 10.1109/TFUZZ.2017.2744605, 2018.

[10] G. Daniello, M. Gaeta, S. Tomasiello, and L. Rarita. A fuzzy consensus approach for group decision making with variable importance of experts. International Conference on Fuzzy Systems (FUZZ-IEEE), Beijing, China, pages 1693-1700, 2016.

[11] G. Deffuant, D. Neau, F. Amblard, and G. Weisbuch. Mixing beliefs among interacting agents. Advances in Complex Systems, 3(01n04):87-98, 2000.

[12] M. J. del Moral, J.M. Tapia, F. Chiclana, and E. Herrera-Viedma. A comparative study on consensus measures in group decision making. International Journal of Intelligent Systems, Doi: 10.1002/int.21954, 2018.

[13] Q. Dong and O. Cooper. A peer to peer dynamic adaptive consensus reaching model for the group ahp decision making. European Journal of Operational Research, 250:521-530, 2016.

[14] Y.C. Dong, X. Chen, H. Liang, and C. Li. Dynamics of linguistic opinion formation in bounded confidence model. Information Fusion, 32:52-61, 2016.

[15] Y.C. Dong, H. Zhang, and E. Herrera-Viedma. Consensus reaching model in the complex and dynamic magdm problem. Knowledge-Based Systems, 106(1):206-219, 2016.

[16] Y.C. Dong, H. Zhang, and E. Herrera-Viedma. Integrating experts' weights generated dynamically into the consensus reaching process and its applications in managing noncooperative behaviors. Decision Support Systems, 84(1):1-15, 2016.

[17] M. Fedrizzi, M. Fedrizzi, and R.A. Pereira. Soft consensus and network dynamics in group decision making. International Journal of Intelligent Systems, 14(1):63-77, 1999.

[18] M. Fedrizzi, M. Fedrizzi, and R.A. Pereira. Consensus modelling in group decision making: dynamical approach based on fuzzy preferences. New Mathematics and Natural Computation, 3(02):219-237, 2007. 
[19] E. Forman and K. Peniwati. Aggregating individual judgments and priorities with the analytic hierarchy process. European journal of operational research, 108(1):165-169, 1998.

[20] M. Gupta and B.K. Mohanty. An algorithmic approach to group decision making problems under fuzzy and dynamic environment. Expert Systems with Applications, 55:118$132,2016$.

[21] R. Hegselmann and U. Krause. Opinion dynamics and bounded confidence models, analysis, and simulation. Journal of Artificial Societies and Social Simulation, 5(3), 2002.

[22] E. Herrera-Viedma, S. Alonso, F. Chiclana, and F. Herrera. A consensus model for group decision making with incomplete fuzzy preference relations. IEEE Transactions on Fuzzy Systems, 15(5):863-877, 2007.

[23] J. Kacprzyk. Group decision making with a fuzzy linguistic majority. Fuzzy Sets and Systems, 18:105-118, 1986.

[24] J. Kacprzyk and S. Zadrożny. Soft computing and web intelligence for supporting consensus reaching. Soft Computing, 14(8):833-846, 2010.

[25] B. Kitchenham, O. Brereton, D. Budgen, M. Turner, J. Bailey, and S. Linkman. Systematic literature reviews in software engineering-a systematic literature review. Information and software technology, 51(1):7-15, 2009.

[26] Patrick R Laughlin. Social choice theory, social decision scheme theory, and group decision-making. Group Processes 83 Intergroup Relations, 14(1):63-79, 2011.

[27] D. Li, W. Zeng, and L. Li. Fuzzy group decision making based on variable weigthed averaging operators. International Conference on Fuzzy Systems (FUZZ-IEEE), Beijing, China, pages 1416-1421, 2014.

[28] G. Li, G. Kou, and Y. Peng. Dynamic fuzzy multiple criteria decision making for performance evaluation. Technological and Economic Development of Economy, 21(5):705$719,2015$.

[29] H. Li, L. Li, J. Wang, Z. Mo, and Y. Li. Fuzzy decision making based on variable weights. Mathematical and computer Modelling, 39(2-3):163-179, 2004.

[30] W. Liu, Y. Dong, F. Chiclana, F.J. Cabrerizo, and E. Herrera-Viedma. Group decisionmaking based on heterogeneous preference relations with self-confidence. Fuzzy Optimization and Decision Making, 16(4):429-447, 2017.

[31] R. Lourenzutti and R. Krohling. A generalized topsis method for group decision making with heterogeneous information in a dynamic environment. Information Sciences, 330(1):1-18, 2016. 
[32] P. Luukka, M. Collan, and M. Fedrizzi. A dynamic fuzzy consensus model with random iterative steps. In System Sciences (HICSS), 2015 48th Hawaii International Conference on, pages 1474-1482. IEEE, 2015.

[33] F. Mata, L. Martínez, and E. Herrera-Viedma. An adaptive consensus support model for group decision making problems in a multi-granular fuzzy linguistic context. IEEE Transactions on Fuzzy Systems, 17(2):279-290, 2009.

[34] I. Palomares, L. Martinez, and F. Herrera. Mentor: a graphical monitoring tool of preferences evolution in large-scale group decision making. Knowledge-Based Systems, 58:66-74, 2014.

[35] R.O. Parreiras, P. Ekel, and F. Bernardes. A dynamic consensus scheme based on a nonreciprocal fuzzy preference relation modeling. Information Sciences, 211:1-17, 2012.

[36] R.O. Parreiras, P. Ekel, J.S.C Martini, and R.M. Palhares. A flexible consensus scheme for multicriteria group decision making under linguistic assessments. Information Sciences, 180(7):1075-1089, 2010.

[37] D. Peng and H. Wang. Dynamic hesitant fuzzy aggregation operators in multi-period decision making. Kybernetes, 43(5):715-736, 2014.

[38] I.J. Pérez, F.J. Cabrerizo, S. Alonso, and E. Herrera-Viedma. A new consensus model for group decision making problems with non homogeneous experts. IEEE Transactions on Systems, Man, and Cybernetics: Systems, 44(4):494-498, 2014.

[39] I.J. Pérez, F.J. Cabrerizo, and E. Herrera-Viedma. A mobile decision support system for dynamic group decision making problems. IEEE Transactions on Systems, Man and Cybernetics - Part A: Systems and Humans, 40(6):1244-1256, 2010.

[40] I.J. Pérez, F.J. Cabrerizo, and E. Herrera-Viedma. Group decision making problems in a linguistic and dynamic context. Expert Systems with Applications, 38(3):1675-1688, 2011.

[41] I.J. Pérez, F.J. Cabrerizo, and E. Herrera-Viedma. A mobile group decision making model for heterogeneous information and changeable decision contexts. International Journal of Uncertainty, Fuzziness and Knowledge-Based Systems, 19(1):33-52, 2011.

[42] R. Ramanathan and L.S. Ganesh. Group preference aggregation methods employed in ahp: An evaluation and an intrinsic process for deriving members' weightages. European Journal of Operational Research, 79(2):249-265, 1994.

[43] T.L. Saaty. Fundamentals of decision making and priority theory with the analytic hierarchy process, volume 6. Rws Publications, 2000.

[44] J. Wu and F. Chiclana. Visual information feedback mechanism and attitudinal prioritisation method for group decision making with triangular fuzzy complementary preference relations. Information Sciences, 279:716-734, 2014. 
[45] J. Wu, F. Chiclana, H. Fujita, and E. Herrera-Viedma. A visual interaction consensus model for social network group decision making with trust propagation. KnowledgeBased Systems, 122:39-50, 2017.

[46] Z. Xu. On multi-period multi-attribute decision making. Knowledge-Based Systems, 21(2):164-171, 2008.

[47] Z. Xu. A method based on the dynamic weighted geometric aggregation operator for dynamic hybrid multi-attribute group decision making. International Journal of Uncertainty, Fuzziness and Knowledge-Based Systems, 17(1):15-33, 2009.

[48] Z. Xu. Multi-period multi-attribute group decision-making under linguistic assessments. International journal of general systems, 38(8):823-850, 2009.

[49] Z. Xu and R.R. Yager. Dynamic intuitionistic fuzzy multi-attribute decision making. International Journal of Approximate Reasoning, 48(1):246-262, 2008.

[50] S. Zadrożny and J. Kacprzyk. An internet-based group decision and consensus reaching support system. In Applied decision support with soft computing, pages 263-276. Springer, 2003. 